\title{
Toward neuro-inspired computing using a small network of micro-ring resonators on an integrated photonic chip
}

\author{
Florian Denis-le Coarer ${ }^{\mathrm{a}}$, Damien Rontani ${ }^{\mathrm{a}}$, Andrew Katumba ${ }^{\mathrm{b}}$, Matthias Freiberger ${ }^{\mathrm{c}}$, Joni \\ Dambre $^{\mathrm{c}}$, Peter Bienstman ${ }^{\mathrm{b}}$, and Marc Sciamanna ${ }^{\mathrm{a}}$ \\ ${ }^{a}$ Chair in Photonics, LMOPS EA 4423 laboratory, CentraleSupelec,Univ. of Paris-Saclay, and \\ Univ. of Lorraine, 2 Rue Edouard Belin, 57070 Metz, France \\ ${ }^{\mathrm{b}}$ Photonic research group, Ghent University - IMEC, Technologiepark Zwijnaarde 15, 9052 \\ Ghent, Zwinjnaarde, Belgium \\ c IDLab, Ghent University - IMEC, Technologiepark Zwijnaarde 15, 9052 Ghent, Zwinjnaarde, \\ Belgium
}

\begin{abstract}
We present in this work numerical simulations of the performance of an on-chip photonic reservoir computer using nonlinear microring resonator as neurons. We present dynamical properties of the nonlinear node and the reservoir computer, and we analyse the performance of the reservoir on a typical nonlinear Boolean task : the delayed XOR task. We study the performance for various designs (number of nodes, and length of the synapses in the reservoir), and with respect to the properties of the optical injection of the data (optical detuning and power). From this work, we find that such a reservoir has state-of-the art level of performance on this particular task - that is a bit error rate of $2.510^{-4}$ - at $20 \mathrm{~Gb} / \mathrm{s}$, with very good power efficiency (total injected power lower than $1.0 \mathrm{~mW}$ ).
\end{abstract}

Keywords: Neuromorphic Computing, Reservoir Computing, Integrated Photonics, Microring Resonators

\section{INTRODUCTION}

In recent years, the field of data processing has faced new challenges related to the ability of systems to process large amounts of data at high-speed rates with good energy efficiency. ${ }^{1}$ These issues can be addressed through the implementation, at the physical layer, of machine learning techniques. One of them is called reservoir computing : this is a supervised technique of machine learning that appeared a decade ago $^{2-4}$ as a promising paradigm to implement artificial neural network at a hardware level. Among existing hardware implementations, ${ }^{5-8}$ reservoir computing has been already tested experimentally using photonic components, ${ }^{9-22}$ hence leading the field of photonic reservoir computing as a good candidate for high-speed, energy-efficient data processing.

We analyse here the performances of a novel architecture for reservoir computing : a small network of nonlinear microring resonators integrated on a silicon photonic chip. We use the so-called swirl topology, previously used in a reservoir architecture made of passive elements, ${ }^{20}$ specifically waveguides, splitters, and combiners. It has been demonstrated that this topology represents a good compromise between wave mixing in a planar structure and losses inherent to integrated interconnections. Here, we simply replace in our reservoir the passive nodes by nonlinear microring resonators, thus introducing nonlinearities and dynamical behaviour directly in the recurrence of the reservoir.

Recently, the dynamical properties of Silicon-On-Insulator (SOI) microring resonators have attracted a lot of attention lately. ${ }^{23-29}$ This integrated element is mostly used as an optical filter, ${ }^{30}$ but can also be integrated in more complex structures to attain all-optical information processing, like classical boolean tasks, ${ }^{31}$ optical thresholding, ${ }^{32}$ pulse restoration, ${ }^{33}$ or ASK-to-PSK generation. ${ }^{34}$

Further author information: (Send correspondence to Florian Denis-le Coarer, or Damien Rontani)

Florian Denis-le Coarer : E-mail : florian.denis@centralesupelec.fr

Damien Rontani : E-mail : damien.rontani@centralesupelec.fr 
We analyse through numerical simulations the performances of such a system on a Boolean nonlinear task: the delayed XOR task. We perform a parametric study on the reservoir in terms of the waveguide length between consecutive nodes, the input power, and the optical detuning, which is the frequency difference between the injected light and the resonance frequency of a micro-ring resonator. The level of performance is quantified using the bit error rate (BER), which is the number of errors made by the reservoir divided by the total number of injected bits. We report on state-of-the-art performances on this particular task, ${ }^{20}$ with a typical BER lower than $10^{-3}$ for a large set of injection parameters (i.e. injected power and optical detuning). Besides, this analysis points out also the energy efficiency of the proposed reservoir architecture, indeed the total optical input power needed to perform this task is less than $2.5 \mathrm{~mW}$.

This paper is organised as follows. First we introduce the models of the reservoir computer, and the nonlinear microring resonator. Then we analyse the dynamical properties of a single node, and the $4 \times 4$ reservoir. Finally, we study through numerical simulations the performance of the reservoir depending on its design and injection parameters.

\section{MODEL}
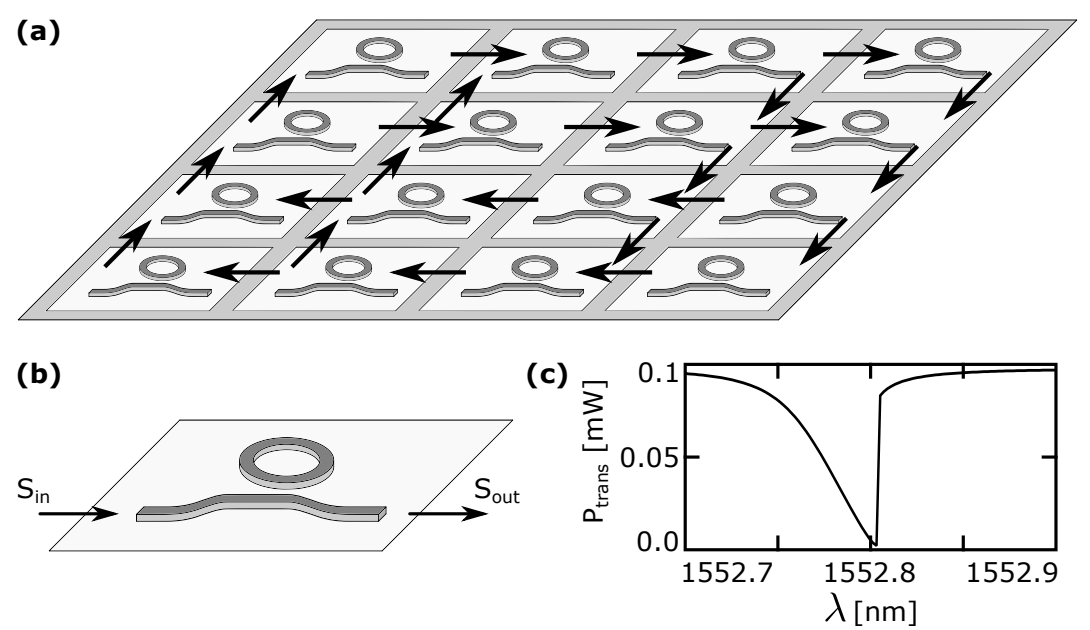

Figure 1. (a) Scheme of the reservoir computer : a $4 \times 4$ swirl network with nonlinear micoring resonators as nodes. (b) Scheme of a nonlinear microring resonator, with a typical $4.0 \mu \mathrm{m}$-radius. (c) Transmission curve of a nonlinear microring resonator. Such a componant can be used as a filter.

\subsection{On-chip reservoir computer}

We present in Fig. 1(a) a schematic view of the integrated photonic reservoir under consideration in this work. The reservoir has a $4 \times 4$ swirl topology, each node - or neuron - is a nonlinear microring resonator (see Subsection 2.2), and the connections between consecutive nodes - or synapses - are ensured by waveguides with typical losses of $3.0 \mathrm{~dB} / \mathrm{cm}$. The swirl topology, introduced in Ref $20,{ }^{20}$ satisfies the planarity of the integrated photonic reservoir while it allows a reasonable mixing of the input signals.

The reservoir model is given by Eqs. (1)-(2) (respectively the state update of the reservoir and the output of the reservoir), where bold variables are either vectors or matrices :

$$
\begin{aligned}
\mathbf{x}[k+1] & =f\left(\mathbf{x}[k], \mathbf{W}_{\text {res }} \mathbf{x}[k]+\mathbf{W}_{\text {in }} \mathbf{u}[k+1]\right), \\
\mathbf{y}_{\text {out }}[k] & =\mathbf{W}_{\text {out }} \mathbf{x}_{\text {detector }}[k],
\end{aligned}
$$

where $\mathbf{x}$ is the state of the reservoir, $\mathbf{x}_{\text {detector }}$ the state of the reservoir after the detector, $\mathbf{y}$ the output signal of the reservoir, $\mathbf{u}$ the input signal in the reservoir, $f$ a nonlinear vector field that describes the nonlinear behaviour of the reservoir. $\mathbf{W}_{\text {in }}, \mathbf{W}_{\text {res }}, \mathbf{W}_{\text {out }}$ are respectively the input matrix that accounts for the input weights to the 
reservoir, the interconnection matrix that represents the connections between each node in the reservoir, and the readout matrix. $\mathbf{W}_{\text {out }}$ accounts for the output weights of the reservoir and is determined through the training procedure.

In our simulations, as we inject the same input stream on all active nodes, the input matrix $\mathbf{W}_{\text {in }}$ is the identity matrix. The interconnection matrix $\mathbf{W}_{\text {res }}$ takes into account the splitting ratios, the losses, and random phase shifts uniformly distributed on $[-\pi, \pi]$. Finally, we use in our simulations a realistic photodetector model taking into account the detector bandwidth as a low-pass filter with $3 \mathrm{~dB}$ cutoff at $25 \mathrm{GHz}$, and various noise contributions (including shot and thermal noise). ${ }^{32}$

\subsection{Single node : nonlinear microring resonator}

In this section, we give the model of the nonlinear microring resonator used as a building block in our reservoir computer. Figure 1(b) depicts a scheme of a SOI microring resonator. This integrated component has been widely described in $^{23,24}$ using its coupled-mode theory (CMT) model. A microring resonator is described in the CMT framework by its input/output relation (given in Eq. (3)), where $s_{i n}$ (resp. $s_{\text {out }}$ ) is the input signal (resp. output signal)* and three states variables : $a$ the complex mode amplitude in the cavity - see Eq. (4) - with $|a|^{2}$ the energy in the cavity, $N$ the concentration of free carriers (see Eq (5)), and $\Delta T$ the temperatures variations which variations are depicted in Eq. (6). The full model reads :

$$
\begin{aligned}
s_{\text {out }} & =e^{j \phi_{c}} s_{\text {in }}+\kappa a, \\
\frac{\mathrm{d} a}{\mathrm{~d} t} & =\left[j\left(\omega_{r}+\delta \omega_{n l}-\omega\right)-\frac{\gamma_{\text {loss }}}{2}\right] a+\kappa s_{\text {in }}, \\
\frac{\mathrm{d} \Delta T}{\mathrm{~d} t} & =-\frac{\Delta T}{\tau_{t h}}+\frac{\Gamma_{t h} \gamma_{a b s}|a|^{2}}{\rho_{S i} c_{p, S i} V_{t h}}, \\
\frac{\mathrm{d} N}{\mathrm{~d} t} & =-\frac{N}{\tau_{f c}}+\frac{\Gamma_{F C A} \beta_{S i} c^{2}|a|^{4}}{2 \hbar \omega V_{F C A}^{2} n_{g}^{2}},
\end{aligned}
$$

where $\omega=2 \pi c \lambda$ is the optical pulsation of the injected light, $\omega_{r}=2 \pi c / \lambda_{r}$ is the resonance pulsation of the nonlinear microring resonator, $\delta \omega_{n l}$ is a nonlinear optical detuning that depends on the variations of the temperature $\Delta T$ and the amount of free carriers $N, \gamma_{\text {loss }}\left(|a|^{2}, N\right)$ is the total loss in the cavity due to imperfect coupling, radiations, and absorption. $\kappa$ is the coupling coefficient between the ring resonator and the waveguide.

The other parameters are imposed by the design of the microring, and in particular the material properties. $\tau_{t h}$ and $\tau_{f c}$ are the relaxation times respectively associated to the temperature variations and the free carriers. $c_{p, S i}$ is the thermal capacity of the silicon, $\beta_{S i}$ the constant describing the two-photons absorption in the silicon, $n_{S i}$ is the refractive index of the bulk silicon, and $\rho_{S i}$ the density of the silicon. We also define the effective volumes and confinements for the nonlinear effects (two-photons absorption (TPA) and free carrier absorption $(F C A)): \Gamma_{T P A}, V_{T P A}, \Gamma_{F C A}$, and $V_{F C A}$. See Ref. $24^{24}$ for a more detailed description of the model, and note that we use the same numerical values of the parameters.

Figure 1(c) shows the transmission curve of a nonlinear microring resonator using the model presented above, with a resonance wavelength $\lambda_{r}=1552.77 \mathrm{~nm}$. This transmission curve presents an asymmetry in the resonance of the microring resonator, due to nonlinear effect TPA and FCA. ${ }^{24}$ This figure also shows how such an integrated element can be used as a notch optical filter, since a very specific band of frequency is cut off.

\section{DYNAMICAL PROPERTIES}

We present in this section dynamical results for (i) a nonlinear microring resonator as a single element, and (ii) a $4 \times 4$ swirl network of integrated ring resonators.

\footnotetext{
${ }^{*}\left|s_{\text {in }}\right|^{2}$ and $\left|s_{\text {out }}\right|^{2}$ are respectively the input and output power in the microring resonator.
} 


\subsection{Nonlinear microring resonator}

Silicon-on-Insulator microring resonators are nonlinear integrated components used mainly as optical notch filters (see Fig. 1(c)), but they also have rich nonlinear behaviours. They can show in particular oscillations (see Fig. 2(f)) or excitability, ${ }^{23,24}$ depending on the input parameters. As for any optical injection problem, the parameters of interest are the input power $P_{i n}=\left|s_{i n}\right|^{2}$, and the optical detuning $\delta \lambda$ that is in our model the wavelength difference between the injected light and the resonance of the microring $\delta \lambda=\lambda-\lambda_{r}$.
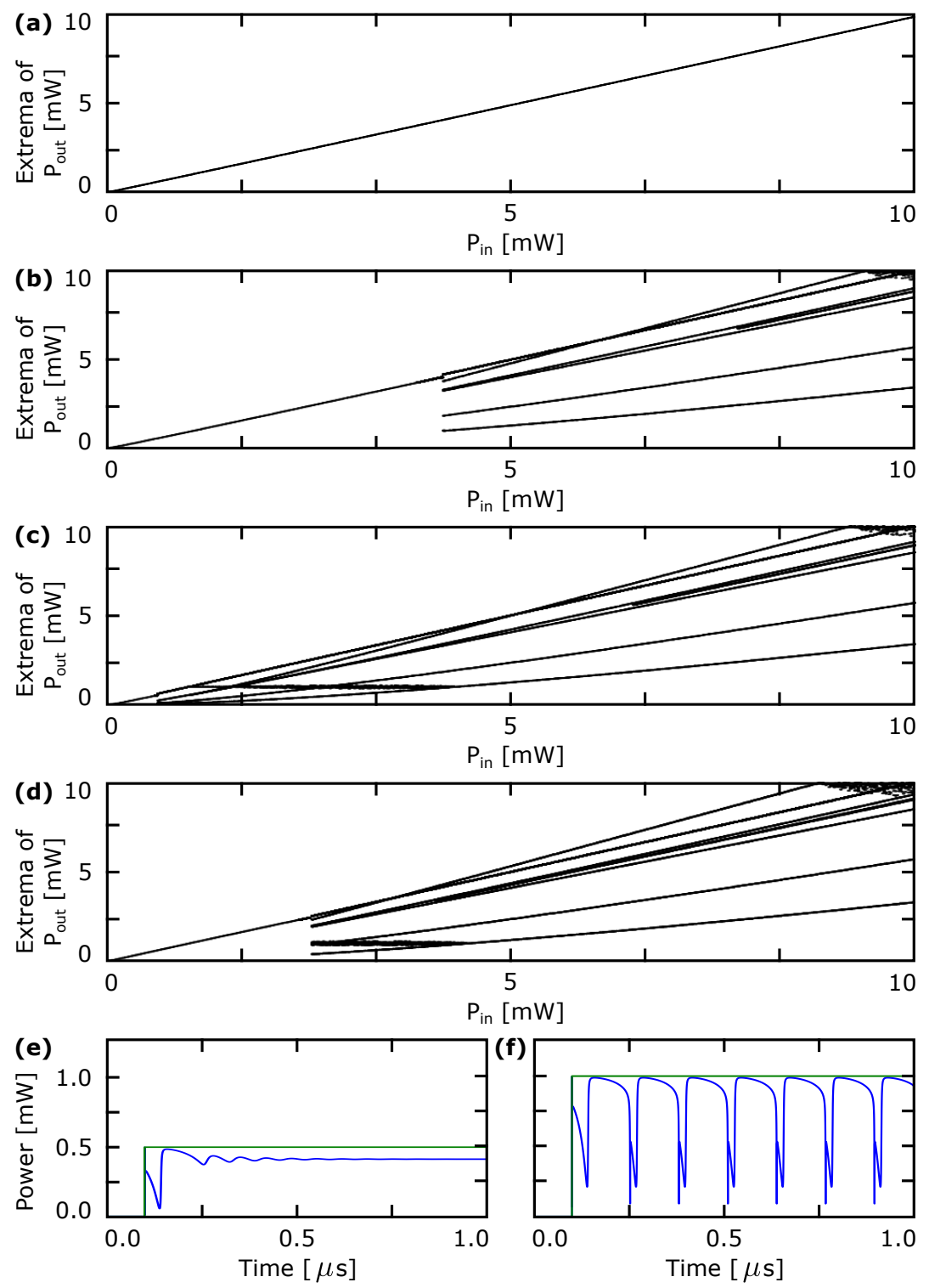

Figure 2. (a)-(d) Bifurcation diagrams of a nonlinear microring resonator for various values of the optical detuning : $\delta \lambda=-50 \mathrm{pm}, \delta \lambda=-20 \mathrm{pm}, \delta \lambda=0 \mathrm{pm}$, and $\delta \lambda=100 \mathrm{pm}$ respectively. (e)-(f) Time series of a nonlinear microring resonator for (e) : $\delta \lambda=0 \mathrm{pm}, P_{i n}=0.5 \mathrm{~mW}$ (steady-state), and (f) $: \delta \lambda=0 \mathrm{pm}, P_{i n}=1.0 \mathrm{~mW}$ (self-pulsation).

We perform a more in-depth analysis of the dynamics of a single nonlinear microring by plotting in Fig. 2(a)(d) bifurcation diagrams of a ring resonator for various values of the optical detuning. These bifurcation diagrams are obtained as follows : the CMT-model of a single, uncoupled, nonlinear microring resonator subjected to power steps (between $P_{i n, 0}=0$, and various values of $P_{i n, 1}$ ) is integrated using the Euler method, with a $1.0 \mathrm{ps}$ 
integration time step, a $10.0 \mathrm{ps}$ sampling time (for graphical representation), and over a total duration of $2.5 \mu \mathrm{s}$. For each value of the bifurcation parameter, here $P_{i n, 1}$ the high value of the power step, we extract from the time series the consecutive extrema of the output power (after deleting the transient). Hence the system is on a steady-state if there is only one point, and the system is oscillating if there are two or more points.

Figures 2(a)-(d) show bifurcation diagrams of an uncoupled ring resonator for respectively $\delta \lambda=-50 \mathrm{pm}$, $\delta \lambda=-20 \mathrm{pm}, \delta \lambda=0 \mathrm{pm}$, and $\delta \lambda=100 \mathrm{pm}$. For $\delta \lambda=-50 \mathrm{pm}$, the microring is stable for any injection. For other values of the optical detuning, the system can be both on a steady state or self pulsating depending on the high value of the power step. For $\delta \lambda=-20 \mathrm{pm}$, the microring oscillates if $P_{i n, 1}>3.5 \mathrm{~mW}$, for $\delta \lambda=0 \mathrm{pm}$, the microring oscillates if $P_{i n, 1}>0.54 \mathrm{~mW}$, and for $\delta \lambda=100 \mathrm{pm}$, the microring oscillates if $P_{i n, 1}>2.3 \mathrm{~mW}$.

We give in Figs. 2(e)-(f) examples of time series of a single nonlinear microring resonator for $\delta \lambda=0 \mathrm{pm}$ and respectively $P_{i n, 1}=0.5 \mathrm{~mW}$ and $P_{i n, 1}=1.0 \mathrm{~mW}$. The microring is on a steady state for $P_{i n, 1}=0.5 \mathrm{~mW}$, and is oscillating for $P_{i n, 1}=1.0 \mathrm{~mW}$, as expected from Fig. 2(c). Figure. 2(e) shows the richness of the transient before the steady-state, which is interesting from a reservoir computing perspective.

\section{$3.24 \times 4$ network}
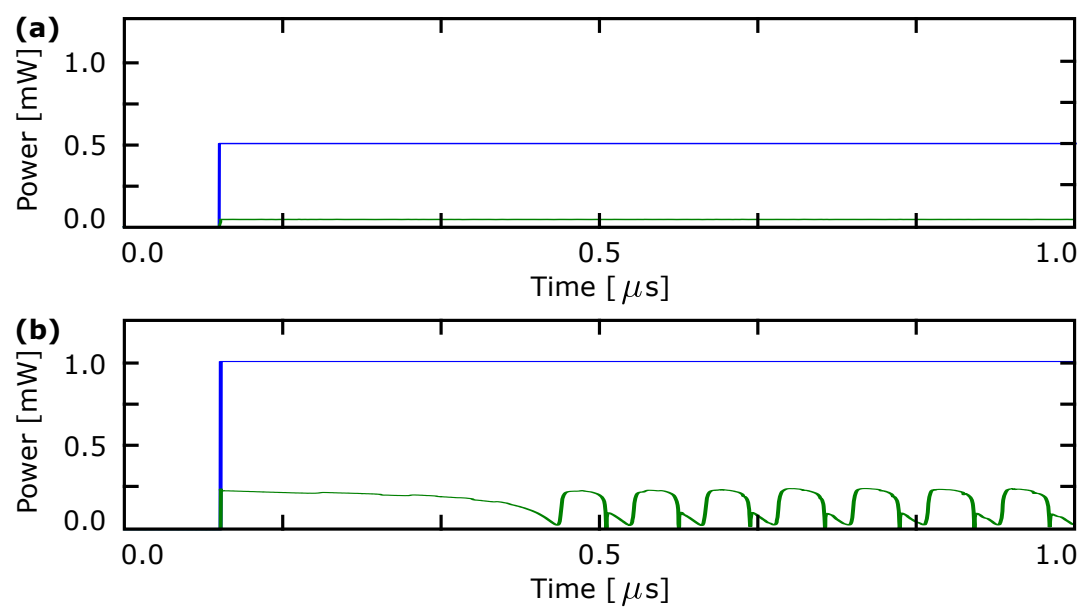

Figure 3. Time series at one output of the reservoir. Each of the 16 nodes is injected with the same input, that is a power step. Injection parameters are the same than in Fig. 2(e)-(f). (a) $\delta \lambda=0 \mathrm{pm}, P_{\text {in }}=0.5 \mathrm{~mW}$ (steady-state), and (b) $\delta \lambda=0 \mathrm{pm}, P_{i n}=1.0 \mathrm{~mW}$ (self-pulsation).

We present in Fig. 3 time series at the output of the reservoir. The power step (blue curve) is injected on all nodes, and the output of one ring after the detector is recorded and plotted in green. The injection parameters are respectively the same than in figures 2(e)-(f). Hence we report a steady-state in Fig.3(a) for $\delta \lambda=0 \mathrm{pm}$, $P_{i n}=0.5 \mathrm{~mW}$, and self-pulsation in Fig. $3(\mathbf{b})$ for $\delta \lambda=0 \mathrm{pm}, P_{i n}=1.0 \mathrm{~mW}$. We also see that the output is attenuated due to the $3.0 \mathrm{~dB}$ loss at each of the power splitters, hence the output power is divided by four since there are two splitters between the output of the ring and the detector.

We present in this section the performance of the reservoir computer for various design of the reservoir. The reservoir performance is measured using the Bit Error Rate (BER), that is the number of errors made by the reservoir divided by the total number of tested bits. Typically, a BER of $10^{-3}$ is acceptable and can be used in telecommunication applications providing the additional use of error correction codes.

\subsection{Influence of the number of nodes}

In this section, we present the performance of the reservoir for various designs in terms of number of nodes in the reservoir. Typically, we use numerical simulations to measure the performance of the reservoir when we increase the number of nodes in the square swirl reservoir $(1 \times 1,2 \times 2,3 \times 3,4 \times 4$, and $5 \times 5$ reservoirs $)$. We inject the same input stream - 20,000 randomly chosen bits - on all nodes, with an optical detuning $\delta \lambda=50 \mathrm{pm}$, a power 
modulation at $20 \mathrm{~Gb} / \mathrm{s}$ between $P_{i n, 0}=0.0 \mathrm{~mW}$ and $P_{i n, 1}=0.3 \mathrm{~mW}$, and an interdelay of $31.25 \mathrm{ps}$, which represent the length of the interconnection waveguide. These injection parameters are chosen as they give good performance of the 16-nodes reservoir (see Fig. 5(b) in subsection 3.4). The training of the reservoir is done using a regularized ridge regression on $16,000 \mathrm{bits}$, and the testing is done on the 4,000 remaining bits.
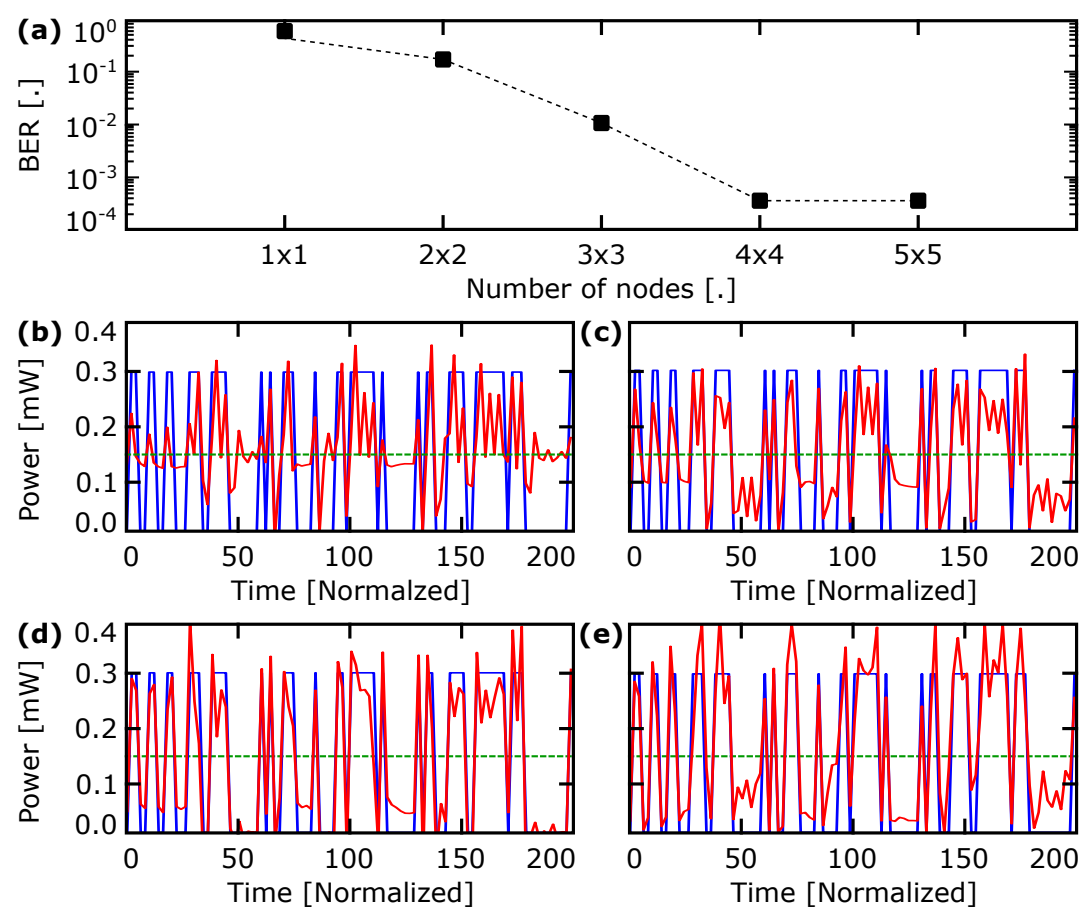

Figure 4. (a) Performance of the reservoir as a function of the number of nodes for a squared reservoir. The task is the delayed XOR. The data is injected on all nodes, with a power modulation at $20 \mathrm{~Gb} / \mathrm{s}$ between $P_{i n, 0}=0.0 \mathrm{~mW}$ and $P_{i n, 1}=0.3 \mathrm{~mW}$, with an optical detuning $\delta \lambda=50 \mathrm{pm}$, and a reservoir interdelay of $31.25 \mathrm{ps}$. (b)-(e) Labels (blue), threshold (green), and down-sampled output of the trained reservoir (red), for (b) the $2 \times 2$ reservoir, (c) the $3 \times 3$ reservoir, (d) the $4 \times 4$ reservoir, and (e) the $5 \times 5$ reservoir.

The results are depicted in Fig. 4(a). The performance of the reservoir is acceptable if the number of nodes in the neural network is higher than 16 , and we measure the best measurable performance $2.5 \times 10^{-4}$ for both a $4 \times 4$ and a $5 \times 5$ reservoir. In the following section, we use the $4 \times 4$ swirl topology in order to reduce the simulation time.

Figures 4(b)-(e) shows the target data (in blue), the decision threshold (in dashed green), and the downsampled output of the trained reservoir (in red) for respectively the 4-nodes, the 9-nodes, the 16-nodes, and the 25 -nodes reservoirs. We see in these figures how well the reservoir performs.

\subsection{Reservoir interdelay and mapping of the performance}

In this section, we study the influence of three parameters : the reservoir interdelay that is linked to the intrinsic design of the reservoir through the length of the synapses, the optical detuning, and the high value of the power modulation. The results are given in Fig. 5.

Figure 5(a) gives the performance of the reservoir on the delayed XOR task as a function of the reservoir interdelay. In this simulation, all the ring resonators of the reservoir are identical. The simulations parameters are identical to those of Fig. 4, and we train/test the reservoir with in the same conditions. The figure shows that the reservoir can perform at best for various designs in terms of interconnection length.

We present in Fig. 5(b) a mapping of the performance of the reservoir for various optical detuning and high values of the power modulation. For all the simulations, the design of the reservoir if fixed with an interdelay of 


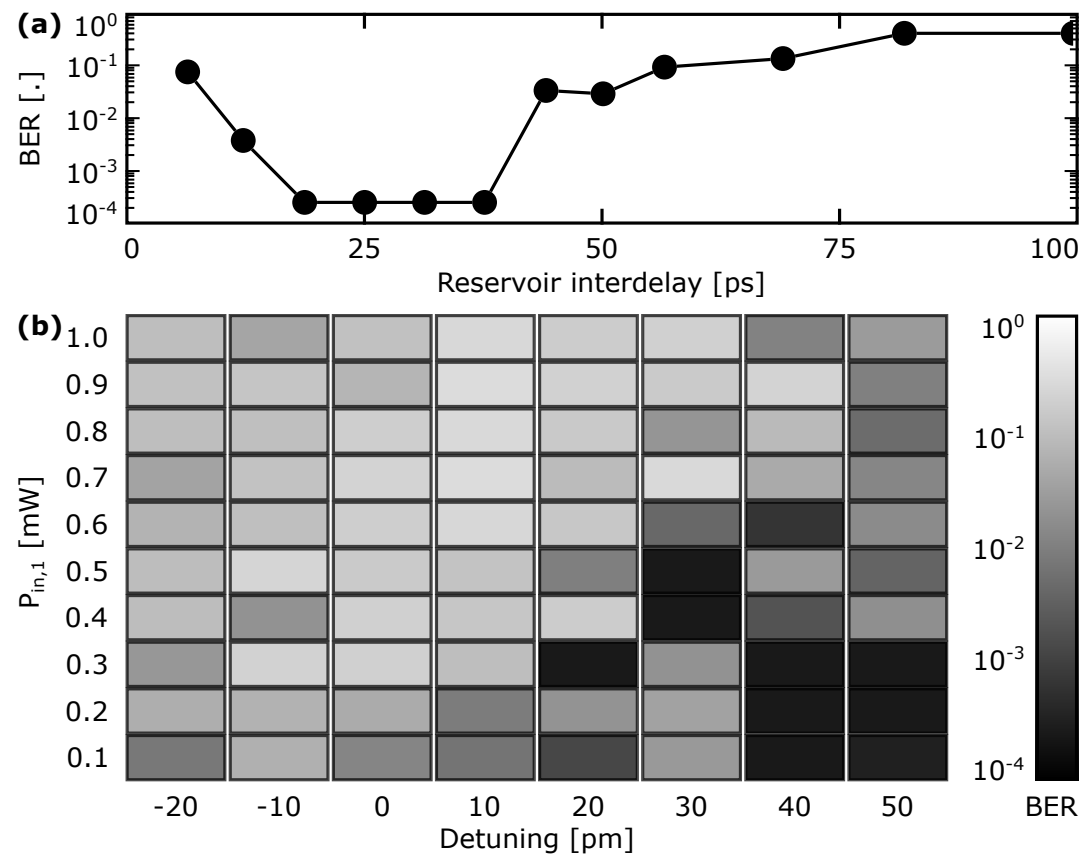

Figure 5. (a) Performance of the reservoir computer as a function of the reservoir interdelay on the delayed XOR task. The data is injected on all nodes, with a power modulation at $20 \mathrm{~Gb} / \mathrm{s}$ between $P_{i n, 0}=0.0 \mathrm{~mW}$ and $P_{i n, 1}=0.3 \mathrm{~mW}$, with an optical detuning $\delta \lambda=50 \mathrm{pm}$. (b) Mapping - in the optical detuning/power modulation plane - of the performance of the reservoir computer on the delayed XOR task. The interdelay is 18.75 ps. The minimum error rate in (a) and (b) is $2.5 \times 10^{-4}$

18.75 ps. In order to study the robustness of the reservoir, we have introduced heterogeneities in the resonance frequency of the ring resonators. Typically, the resonance frequencies of the 16 microring resonators follow a Gaussian distribution centred at $\lambda_{r}$, with a $10 \mathrm{pm}$ standard deviation, which is a rather pessimistic value with respect to the current technology. For all the simulations, the same input stream of 15,000 bits is fed at $20 \mathrm{~Gb} / \mathrm{s}$ on all 16-nodes of the reservoir. We realise the training on 12,000 bits and the testing on the 3,000 remaining bits.

We see in this map that the reservoir can perform at acceptable levels of performance for various injection parameters, and for low power consumption. Indeed the average power consumption of the reservoir is given by Eq. (7) :

$$
<P_{\text {total }}>=N_{\text {nodes }}<P_{\text {in }}>=\frac{P_{\text {in }, 0}+P_{\text {in }, 1}}{2} N_{\text {nodes }},
$$

where $N_{\text {nodes }}$ is the number of nodes, and $P_{i n, 1}$ the high value of the power modulation. Hence, we can find a set of injection parameters where the reservoir computer performs at best with very low power consumption. For instance for a power modulation between $P_{i n, 0}=0.0 \mathrm{~mW}$ and $P_{i n, 1}=0.1 \mathrm{~mW}$, at $\delta \lambda=50 \mathrm{pm}$, the BER is lower than $10^{-3}$ and the total averaged power is $0.8 \mathrm{~mW}$.

\section{CONCLUSION}

Finally, we have suggested a novel integrated reservoir architecture using nonlinear microring resonators as nodes, that can perform very well on nonlinear Boolean task for various operating parameter conditions. We have also connected the nonlinear dynamical properties of the single node of the reservoir (optical detuning, optical power) to the performances of the reservoir computer. Our results motivate further investigations on the performance of this kind of structures, more specifically by studying the performances on other tasks as time series generation, chaos prediction, or nonlinear-channel equalization, with final objective of integrated, high-speed, energy efficient, and reconfigurable all-optical data processing. 


\section{ACKNOWLEDGMENTS}

M.S., D.R., and F.D. acknowledge the support of the Fondation CentraleSupélec, Préfecture de Région GrandEst, Région Grand-Est, Metz Métropole, Département de la Moselle, AIRBUS GDI Simulation, through the funding of the Chair in Photonics and PIANO project. D.R acknowledges the support of AFOSR (grant No. FA-9550-15-1-0279 and FA9550-17-1-0072). All the authors acknowledge the financial support of the BELSPO IAP P7-35 program Photonics@be and the EU Horizon 2020 PHRESCO Grant (Grant No. 688579).

\section{REFERENCES}

[1] Manyika, J., Chui, M., Brown, B., Bughin, J., Dobbs, R., Roxburgh, C., and Byers, A. H., "Big data: The next frontier for innovation, competition, and productivity," (2011).

[2] Maass, W., Natschläger, T., and Markram, H., "Real-time computing without stable states: A new framework for neural computation based on perturbations," Neural computation 14(11), 2531-2560 (2002).

[3] Jaeger, H. and Haas, H., "Harnessing nonlinearity: Predicting chaotic systems and saving energy in wireless communication," Science 304(5667), 78-80 (2004).

[4] Lukoševičius, M. and Jaeger, H., "Reservoir computing approaches to recurrent neural network training," Computer Science Review 3(3), 127-149 (2009).

[5] Nikolić, D., Haeusler, S., Singer, W., and Maass, W., "Temporal dynamics of information content carried by neurons in the primary visual cortex," 1041-1048 (2007).

[6] Schürmann, F., Meier, K., and Schemmel, J., "Edge of chaos computation in mixed-mode vlsi-a hard liquid," Advances in neural information processing systems , 1201-1208 (2005).

[7] Verstraeten, D., Schrauwen, B., and Stroobandt, D., "Reservoir computing with stochastic bitstream neurons," Proceedings of the 16th annual Prorisc workshop , 454-459 (2005).

[8] Kulkarni, M. S. and Teuscher, C., "Memristor-based reservoir computing," Nanoscale Architectures (NANOARCH), 2012 IEEE/ACM International Symposium on , 226-232 (2012).

[9] Larger, L., Baylón-Fuentes, A., Martinenghi, R., Udaltsov, V. S., Chembo, Y. K., and Jacquot, M., "Highspeed photonic reservoir computing using a time-delay-based architecture: Million words per second classification," Physical Review X 7(1), 011015 (2017).

[10] Brunner, D., Soriano, M. C., Mirasso, C. R., and Fischer, I., "Parallel photonic information processing at gigabyte per second data rates using transient states," Nature communications 4, 1364 (2013).

[11] Appeltant, L., Soriano, M. C., Van der Sande, G., Danckaert, J., Massar, S., Dambre, J., Schrauwen, B., Mirasso, C. R., and Fischer, I., "Information processing using a single dynamical node as complex system," Nature communications 2, 468 (2011).

[12] Duport, F., Schneider, B., Smerieri, A., Haelterman, M., and Massar, S., "All-optical reservoir computing," Optics express 20(20), 22783-22795 (2012).

[13] Larger, L., Soriano, M. C., Brunner, D., Appeltant, L., Gutiérrez, J. M., Pesquera, L., Mirasso, C. R., and Fischer, I., "Photonic information processing beyond turing: an optoelectronic implementation of reservoir computing," Optics express 20(3), 3241-3249 (2012).

[14] Soriano, M. C., Ortín, S., Brunner, D., Larger, L., Mirasso, C. R., Fischer, I., and Pesquera, L., "Optoelectronic reservoir computing: tackling noise-induced performance degradation," Optics express 21(1), 12-20 (2013).

[15] Antonik, P., Hermans, M., Duport, F., Haelterman, M., and Massar, S., "Towards pattern generation and chaotic series prediction with photonic reservoir computers," Proc. of SPIE Vol 9732, 97320B-1 (2016).

[16] Tait, A. N., Zhou, E., de Lima, T. F., Wu, A. X., Nahmias, M. A., Shastri, B. J., and Prucnal, P. R., "Neuromorphic silicon photonics," arXiv preprint arXiv:1611.02272 (2016).

[17] Vandoorne, K., Dambre, J., Verstraeten, D., Schrauwen, B., and Bienstman, P., "Parallel reservoir computing using optical amplifiers," IEEE transactions on neural networks 22(9), 1469-1481 (2011).

[18] Mesaritakis, C., Papataxiarhis, V., and Syvridis, D., "Micro ring resonators as building blocks for an alloptical high-speed reservoir-computing bit-pattern-recognition system," JOSA B 30(11), 3048-3055 (2013). 
[19] Mesaritakis, C., Kapsalis, A., and Syvridis, D., "All-optical reservoir computing system based on ingaasp ring resonators for high-speed identification and optical routing in optical networks," SPIE OPTO , 937033937033 (2015).

[20] Vandoorne, K., Mechet, P., Van Vaerenbergh, T., Fiers, M., Morthier, G., Verstraeten, D., Schrauwen, B., Dambre, J., and Bienstman, P., "Experimental demonstration of reservoir computing on a silicon photonics chip," Nature communications $\mathbf{5}$ (2014).

[21] Katumba, A., Freiberger, M., Bienstman, P., and Dambre, J., "A multiple-input strategy to efficient integrated photonic reservoir computing," Cognitive Computation, 1-8 (2017).

[22] Fiers, M. A. A., Van Vaerenbergh, T., Wyffels, F., Verstraeten, D., Schrauwen, B., Dambre, J., and Bienstman, P., "Nanophotonic reservoir computing with photonic crystal cavities to generate periodic patterns," IEEE transactions on neural networks and learning systems 25(2), 344-355 (2014).

[23] Van Vaerenbergh, T., "All-optical spiking neurons integrated on a photonic chip," PhD Thesis (2014).

[24] Van Vaerenbergh, T., Fiers, M., Mechet, P., Spuesens, T., Kumar, R., Morthier, G., Schrauwen, B., Dambre, J., and Bienstman, P., "Cascadable excitability in microrings," Optics express 20(18), 20292-20308 (2012).

[25] Zhang, L., Fei, Y., Cao, Y., Lei, X., and Chen, S., "Experimental observations of thermo-optical bistability and self-pulsation in silicon microring resonators," JOSA B 31(2), 201-206 (2014).

[26] Pernice, W. H., Li, M., and Tang, H. X., "Time-domain measurement of optical transport in silicon microring resonators," Optics express 18(17), 18438-18452 (2010).

[27] Zhang, L., Fei, Y., Cao, T., Cao, Y., Xu, Q., and Chen, S., "Multibistability and self-pulsation in nonlinear high- q silicon microring resonators considering thermo-optical effect," Physical Review A 87(5), 053805 (2013).

[28] Chen, S., Zhang, L., Fei, Y., and Cao, T., "Bistability and self-pulsation phenomena in silicon microring resonators based on nonlinear optical effects," Optics Express 20(7), 7454-7468 (2012).

[29] Amiri, I. S., Ahsan, R., Shahidinejad, A., Ali, J., and Yupapin, P. P., "Characterisation of bifurcation and chaos in silicon microring resonator," IET Communications 6(16), 2671-2675 (2012).

[30] Hryniewicz, J., Absil, P., Little, B., Wilson, R., and Ho, P.-T., "Higher order filter response in coupled microring resonators," IEEE Photonics Technology Letters 12(3), 320-322 (2000).

[31] Caulfield, H. J., Soref, R. A., and Vikram, C. S., "Universal reconfigurable optical logic with silicon-oninsulator resonant structures," Photonics and Nanostructures-Fundamentals and Applications 5(1), 14-20 (2007).

[32] Kishikawa, H., Kondo, T., Goto, N., and Talabattula, S., "Optical thresholder consisting of two cascaded mach-zehnder interferometers with nonlinear microring resonators," Optical Engineering 56(8), 086101 (2017).

[33] Dumeige, Y., Ghisa, L., and Féron, P., "Integrated all-optical pulse restoration with coupled nonlinear microring resonators," Optics letters 31(14), 2187-2189 (2006).

[34] Tanaram, C., Teeka, C., Jomtarak, R., Yupapin, P., Jalil, M., Amiri, I., and Ali, J., "Ask-to-psk generation based on nonlinear microring resonators coupled to one mzi arm," Procedia Engineering 8, 432-435 (2011). 